\title{
A COMPLETE SYSTEM FOR OPERATION OF A SUPERCONDUCTING MAGNET*
}

\author{
G. Codner" ${ }^{\#}$ M. Comfort, D. Sabol, T. VanDerMark, D. Widger, R. Yaeger, \\ Laboratory for Elementary Particle Physics \\ Cornell University, Ithaca, NY 14853, USA
}

\section{Abstract}

A complete system for operating, protecting and monitoring a superconducting magnet is described. This system is used in CESR (Cornell Electron Storage Ring) at Cornell University's Laboratory for Elementary Particle Physics (LEPP) for the CESR superconducting wigglers, part of the accelerator upgrade in pursuit of the CESR charm physics program known as CESR-c [1].

\section{INTRODUCTION}

A Superconducting (SC) Magnet Operation System (MOS) is described for operating and protecting a SC magnet with energy storage up to 120 kilojoules and current up to 375 amperes. The architecture and type of construction provide a basis for a configurable system with particular attention paid to minimizing manufacturing complexity and cost.

Twelve such systems have been built and installed in CESR to operate twelve SC wigglers, which were installed in order to achieve CESR operational goals for the CESR-c physics program [1]. The wigglers are required for damping and emittance control of the stored beams. Additional spurious effects of the wiggler field are larger beam energy spread and vertical betatron tune sensitivity to field strength. The sensitivity of the vertical tune is the main reason for $0.01 \%$ main winding DC current stability specification (see Table 1).

\section{REQUIREMENTS}

Requirements for any superconducting magnet are:

- DC power supply

- Liquid helium (LHe) supply

- Liquid nitrogen (LN2) supply

- Quench detection and protection

In addition to these basic requirements are:

- Precise current regulation

- Power supply voltage limiting

- LHe level regulation

- LN2 level regulation

- Gaseous helium $(\mathrm{GHe})$ pressure regulation

\footnotetext{
${ }^{*}$ Work supported by the National Science Foundation

\#gcodner@lepp.cornell.edu
}

Magnet power must be removed in the event of:

- Magnet Quench

- Low LHe or LN2 level

- Excessive current or voltage

- Insulation vacuum degradation

- Ground faults

Monitoring is needed for:

- Magnet current

- Magnet voltage

- Cryogenic temperatures

- Cryostat LHe level, LN2 level and GHe pressure

- Alarm status and levels

Table 1: CESR Wiggler SC MOS Capabilities

\begin{tabular}{|l|l|l|}
\hline $\begin{array}{l}\text { Main } \\
\text { Winding } \\
\text { DC Current }\end{array}$ & Rating & Limitation(s) \\
\hline Level & $375 \mathrm{~A}$ & $\begin{array}{l}\text { 1. Power supply } \\
\text { 2. Series IGBT } \\
\text { 3. 4\#0 cable }\end{array}$ \\
\hline Accuracy & $0.1 \%$ & $\begin{array}{l}\text { 1. D/A Reference } \\
\text { 2. Transductor } \\
\text { 3. Resistor }\end{array}$ \\
\hline Stability & $0.01 \%$ & $\begin{array}{l}\text { 1.Transductor } \\
\text { 2. Resistor } \\
\text { 3. D/A Reference }\end{array}$ \\
\hline Stored Energy & $120 \mathrm{~kJ}$ & Dump resistor \\
\hline $\begin{array}{l}\text { Trim } \\
\text { Winding } \\
\text { DC Current }\end{array}$ & Rating & Limitation(s) \\
\hline Level & $10 \mathrm{~A}$ & $\begin{array}{l}\text { 1. Power supply } \\
\text { 2. Magnet leads }\end{array}$ \\
\hline Accuracy & $1 \%$ & Hall sensor \\
\hline
\end{tabular}

\section{ARCHITECTURE}

\section{System Block Diagram}

The SCMOS block diagram for a system meeting these requirements is shown in Figure 1. With the exception of the 4-20mA current-to-pressure transducers and the GHe pressure transducer, all of the electronic components and subassemblies are mounted in a 19-inch roll-around rack. 


\title{
Superconducting Magnet Operation System
}

\author{
to $4-20 \mathrm{~mA}$ \\ current- \\ to-pressure \\ transducers (x3)
}

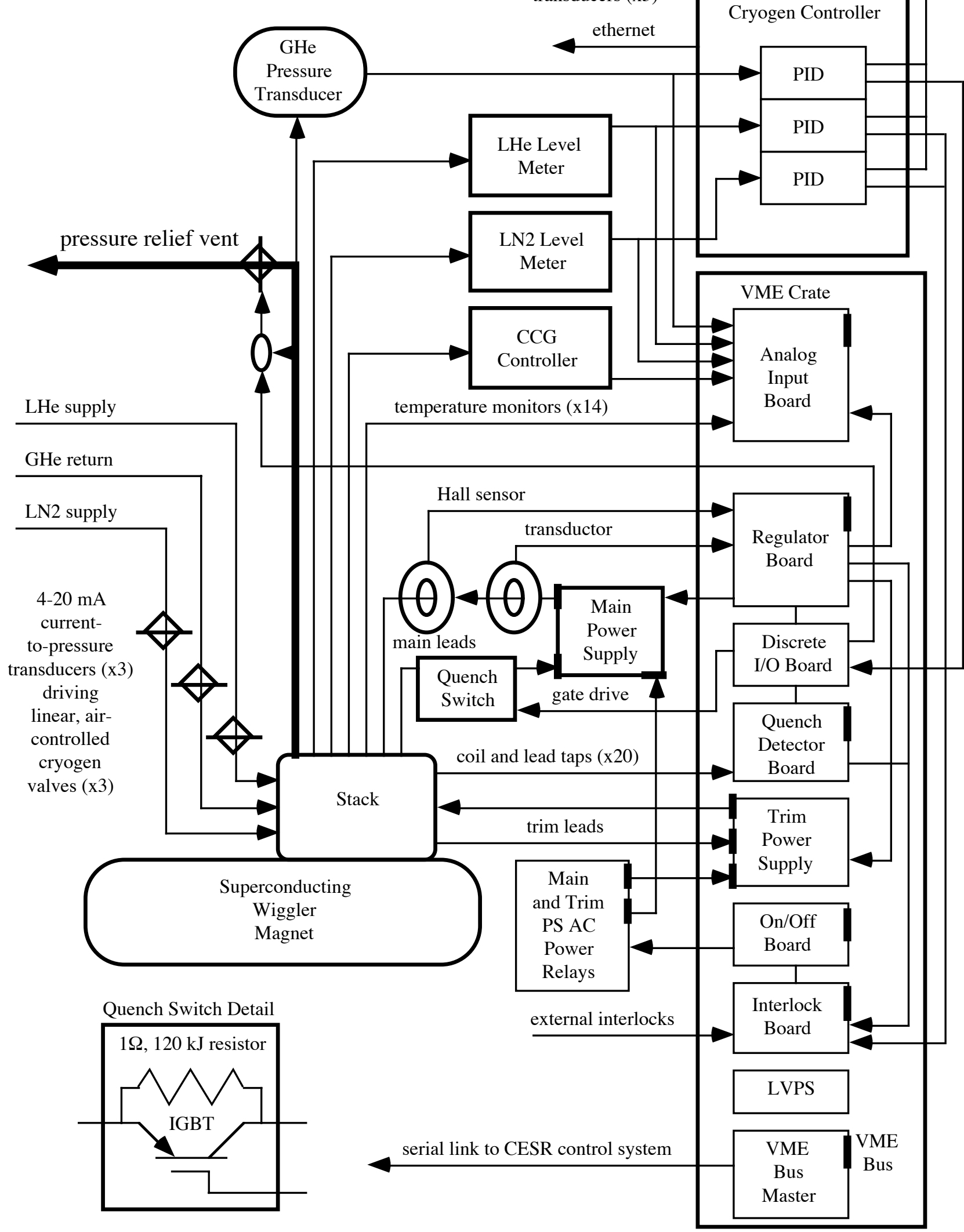

Figure 1: CESR Wiggler Superconducting Magnet Operation System Block Diagram 


\section{Main Power Supply and Current Regulation}

A commercial 3.3 volt, 375 ampere power supply with voltage programming input provides energy to the main windings. To obtain the current regulation accuracy and stability required for CESR-c running, a commercially available 400-ampere transductor is used to sense magnet current for regulation. A Hall-effect current sensor is used as a backup in that both current sensors are used for software current monitoring and hardware overcurrent protection.

The 2000:1 current output of the transductor is fed to a transconductance amplifier on the VME Regulator Board. The transconductance is determined by eight (8), 100 ohm, $0.01 \%$ resistors in series and parallel to make 50 ohms so that 100 milliamperes provides 5 volts directly from the transductor buffer amplifier to the feedback loop input. This method provides a single amplifier stage, thereby providing excellent common-mode rejection while minimizing drift due to time and temperature.

The transductor current signal, converted to a voltage signal proportional to current, is compared with a 16-bit DAC-derived analog reference voltage using a x100 error amplifier and with bandwidth well above the bandwidth of the magnet itself, the latter being used as the dominant pole in the control loop.

\section{Quench Detection and Energy Extraction}

The CESR SC wigglers are protected from quenches internally. Shunt resistors consisting of wound stainless steel wire are in parallel with each winding of the 16 main coil windings, ensuring that the quenching coil absorbs only the energy associated with itself.

Although the wiggler is relatively self-protecting, it is prudent nevertheless to provide quench detection and energy extraction. The magnet current returns through a series IGBT (insulated gate bipolar transistor) in parallel with a one ohm resistor. The IGBT is normally conducting with a voltage drop of approximately 1.7 volts at a current of 141 amperes. A quench is detected by comparing the voltages of the upper and lower halves of the magnet. A difference of 0.5 volts triggers the quench detection circuit which turns off the power supply and removes gate drive from the IGBT. The external resistor absorbs approximately half of the magnet's 25 kilojoules of stored energy.

\section{Trim Power Supply and Current Regulation}

A 5 volt, 10 ampere power supply energizes the trim windings. An external Hall effect current sensor is used to measure the current and an offset error signal is fed back to the remote sense input of the supply.

The trim winding would never quench and would not need protection if it did, however, because of the magnetic coupling between the main and trim windings, protection is required for the trim winding and power supply during a quench in the main coil. A series FET (Field Effect Transistor) and shunt resistor perform this function. The FET is normally driven on and is turned off when a quench is detected.
The trim power supply, quench protection and Hall current sensor are packaged on a custom PCB that plugs into the VME crate.

\section{Cryogenic Controls}

Liquid helium and liquid nitrogen levels are regulated using supply valves for these cryogens. Level meters deliver 4-20 mA signals to a Moore 353 Process Controller. PID (proportional integral derivative) control loops in the controller accept the level inputs and output 4-20 mA valve control signals as appropriate. A third PID control loop looks at helium bath pressure for regulation of that parameter.

The processor controller also provides level faults as relay contact closures to the power supply interlock chain. This controller is separately powered and uses an independent ethernet Modbus link to communicate with the CESR control system,

\section{Interlocks and On/Off Control}

In keeping with long-standing practice in CESR, hardware interlocks and controls are implemented with relays in the spirit of a "C.O. Brown Ready Chain". A string of relay contacts feeds the latched ON relay. An interruption of the chain turns the power supply off.

\section{Packaging}

Most of these functions are provided on 6U-format VME printed circuit boards (PCBs) modules designed inhouse and residing in a VME crate, also assembled inhouse from a VME chassis kit with some modifications. The PCB modules plug into a standard VME backplane for connector P1 and a custom-designed PCB backplane for connector P2. A commercially available industrial controller provides cryogenic controls with closed loop control of LHe level, LN2 level and GHe pressure. The crates are located in the CESR tunnel. Shielding from synchrotron radiation is afforded by placing the crates in a box made of lead-clad copper. The lead is bonded to the copper using automotive body panel adhesive.

\section{ACKNOWLEDGEMENTS}

Thanks go to Margee Carrier, Don Dawson, Gloria LaFave, George Trutt and Peter Quigley for their expertise and technical advice. Thanks to John Sikora, Dave Rice, Charlie Strohman and Mike Billing for software support and integration with the CESR control system. Thanks to the entire dedicated staff of Wilson Synchrotron Laboratory.

\section{REFERENCES}

[1] D. Rice, "CESR-c - A Frontier Machine for QCD and Weak Decay Physics in the Charm Region," EPAC'02, Paris, June 2002, p. 428.

[2] D. Rice, et. al., "Production and Testing Considerations for CESR-c Wiggler Magnets", PAC'03.

[3] Y. He, et. al., "Design and Operation of the Cryostat for the CESR-c Superconducting Wiggler Magnets", PAC'03. 